streptococcal sore-throat) in 33\% of patients, migraine in $6 \%$, and head trauma in $6 \%$. A family history of migraine was elicited in $46 \% ; 5$ parents $(33 \%)$ of affected patients had experienced AWS symptoms. Of 15 patients with follow-up by telephone interview, $20 \%$ had occasional recurrences, $40 \%$ had no further attacks, $40 \%$ were still having symptoms, $4(27 \%)$ developed migraine, and 1 patient $(7 \%)$ had seizures. The interval between initial diagnosis and telephone contact was an average of 6.5 yrs (range, 2.1-13.53 yrs). (Liu AM, Liu JG, Liu Alm GW, Liu GT. "Alice in Wonderland" syndrome: Presenting and follow-up characteristics. Pediatr Neurol 2014 Sep;51(3):317-20).

COMMENTARY. AWS is a disorder of childhood that affects boys more often than girls, and may subsequently 'metamorphose' and develop into migraine in one quarter of patients.

Headache metamorphosing into AWS. Patients with a diagnosis of childhood headache in 1983 were interviewed by telephone in 1993, 2003, and 2013. Of 28 patients monitored, headaches were ongoing in $71 \%$, and distortions of time and space were experienced by $>25 \%$ and $\sim 20 \%$, respectively. There was no clear correlation with migraine, and patients with tension-type headaches also reported the AWS symptoms. Distortions of space and time persist into the fifth decade for many patients initially observed with headaches in childhood [1].

\title{
References.
}

1. Dooley JM, et al. Pediatr Neurol. 2014 Sep;51(3):321-3.

\section{NUTRITIONAL DISORDERS}

\section{THIAMINE DEFICIENCY IN INFANCY}

Investigators at Tel Aviv University, Loewenstein Rehabilitation Hospital, Schneider Children's Medical Center, and other centers in Israel report the clinical presentation of acute encephalopathy in 11 children and the long-term sequelae of 8 who initially survived an episode of thiamine deficiency. In 2003, 20 Israeli infants were seriously affected after being fed an international brand of soy-based formula later found to contain no thiamine. In the acute phase, 6 had bulbar signs, 5 had ophthalmologic signs and 2 had phrenic neuropathy. MRI, the best test for diagnosis of thiamine deficiency in the acute phase, showed symmetric involvement of frontal, temporal and parietal lobes, lesions in the periaqueductal region, thalami, and the mammillary bodies, findings similar to sequelae of hypoxic-ischemic injury. Of 5 patients with cardiac involvement, 3 had cardiomyopathy and died in the acute phase, and one presented with a complete atrioventricular block. Lactic acidosis was present in 10 patients. In long-term follow-up, one patient in a chronic vegetative state died after 6 years, 7 children were mentally retarded and had motor abnormalities, 6 developed severe epilepsy, 3 with West syndrome, 2 had kyphoscoliosis, and one remained in complete A-V block. (MimouniBloch A, Goldberg-Stern H, Strausberg R, et al. Thiamine deficiency in infancy: Longterm follow-up. Pediatr Neurol 2014 Sep;51(3):311-6). 
COMMENTARY. The majority of infants exposed to the thiamine deficient formula in infancy were asymptomatic but a small minority developed encephalopathy and/or cardiomyopathy that were sometimes fatal. Infants who survive thiamine-deficient encephalopathy have a poor prognosis, with motor and cognitive impairment and epilepsy. Thiamine deficiency in a developed country is unusual, but as many as $12.5 \%$ of a population of critically ill Canadian children were found to have significant thiamine deficiency [1]. Wernicke encephalopathy and beriberi during total parenteral nutrition was attributed to multivitamin infusion shortage in a patient with Crohn's disease in California [2]. Almost immediately following intravenous thiamine, the hypotension resolved and the following day she no longer had diplopia, and the ophthalmoplegia had improved. Thiamine deficiency should be considered in patients with malabsorption, malnutrition, and malignancies.

\section{References.}

1. Seear, et al. J Pediatr. 1992 Oct;121(4):533-8.

2. Hahn JS, et al. Pediatrics. 1998 Jan;101(1):E10.

\section{NEUROMUSCULAR DISORDERS}

\section{CONGENITAL MYASTHENIC SYNDROME WITH AGRIN MUTATIONS}

Investigators at Newcastle University, UK, and Hopitaux de Paris, France, report 5 patients from 3 unrelated families with a strikingly homogeneous clinical entity combining congenital myasthenia with distal muscle weakness and atrophy resembling a distal myopathy. MRI and neurophysiological studies were indicative of a mild distal myopathy, but decrement in response to $3 \mathrm{~Hz}$ repetitive nerve stimulation suggested a neuromuscular transmission defect. Post-exercise increment up to $285 \%$ in distal limb muscles was compatible with presynaptic congenital myasthenic syndrome. Immunofluorescence and ultrastructural analyses of muscle end-plate regions showed synaptic remodelling with denervation-reinnervation. Whole-exome sequencing identified five new recessive mutations in the gene encoding agrin. These findings expand the spectrum of congenital myasthenic syndromes due to agrin mutations. (Nicole $\mathrm{S}$, Chaouch A, Torbergsen $\mathrm{T}$, et al. Agrin mutations lead to a congenital myasthenic syndrome with distal muscle weakness and atrophy. Brain 2014 Sep;137(Pt 9):2429-43).

COMMENTARY. The authors recommend examination of patients with apparent distal myopathy for a neuromuscular transmission disorder and for agrin mutations.

Prevalence of congenital myasthenia. The UK prevalence of genetically confirmed congenital myasthenic syndrome (CMS) is 9.2 per million children under 18 years of age. CMS is equally prevalent in girls and boys. CHRNE, RAPSN and DOK7 are the most commonly identified mutations. Prevalence varies across geographical regions in England (2.8 to 14.8 per million). The mean incidence of antibody-positive autoimmune myasthenia was 1.5 per million children per year. Girls were affected more frequently than boys [1]. 